\title{
Fuzzy logic selection as a new reliable tool to identify molecular grade signatures in breast cancer - the INNODIAG study
}

Tatiana Kempowsky-Hamon ${ }^{1,2 \dagger}$, Carine Valle ${ }^{2,3,4 \dagger}$, Magali Lacroix-Triki ${ }^{5}$, Lyamine Hedjazi ${ }^{1,2}$, Lidwine Trouilh²,3,4, Sophie Lamarre 2,3,4 Delphine Labourdette ${ }^{2,3,4}$, Laurence Roger ${ }^{5}$, Loubna Mhamdi ${ }^{5}$, Florence Dalenc ${ }^{6}$, Thomas Filleron ${ }^{7}$, Gilles Favre ${ }^{5}$, Jean-Marie François ${ }^{2,3,4,6}$, Marie-Véronique Le Lann ${ }^{1,2^{*}}$ and Véronique Anton-Leberre $2,3,4$

\begin{abstract}
Background: Personalized medicine has become a priority in breast cancer patient management. In addition to the routinely used clinicopathological characteristics, clinicians will have to face an increasing amount of data derived from tumor molecular profiling. The aims of this study were to develop a new gene selection method based on a fuzzy logic selection and classification algorithm, and to validate the gene signatures obtained on breast cancer patient cohorts.
\end{abstract}

Methods: We analyzed data from four published gene expression datasets for breast carcinomas. We identified the best discriminating genes by comparing molecular expression profiles between histologic grade 1 and 3 tumors for each of the training datasets. The most pertinent probes were selected and used to define fuzzy molecular grade 1-like (good prognosis) and fuzzy molecular grade 3-like (poor prognosis) profiles. To evaluate the prognostic performance of the fuzzy grade signatures in breast cancer tumors, a Kaplan-Meier analysis was conducted to compare the relapse-free survival deduced from histologic grade and fuzzy molecular grade classification.

Results: We applied the fuzzy logic selection on breast cancer databases and obtained four new gene signatures. Analysis in the training public sets showed good performance of these gene signatures for grade (sensitivity from $90 \%$ to $95 \%$, specificity $67 \%$ to $93 \%$ ). To validate these gene signatures, we designed probes on custom microarrays and tested them on 150 invasive breast carcinomas. Good performance was obtained with an error rate of less than $10 \%$. For one gene signature, among 74 histologic grade 3 and 18 grade 1 tumors, 88 cases (96\%) were correctly assigned. Interestingly histologic grade 2 tumors $(n=58)$ were split in these two molecular grade categories.

Conclusion: We confirmed the use of fuzzy logic selection as a new tool to identify gene signatures with good reliability and increased classification power. This method based on artificial intelligence algorithms was successfully applied to breast cancers molecular grade classification allowing histologic grade 2 classification into grade 1 and grade 2 like to improve patients prognosis. It opens the way to further development for identification of new biomarker combinations in other applications such as prediction of treatment response.

Keywords: Breast cancer, Molecular grade, Gene signatures, Fuzzy logic

\footnotetext{
* Correspondence: mvlelann@laas.fr

†Equal contributors

${ }^{1}$ CNRS, LAAS, F-31400 Toulouse, France

2Université de Toulouse; INSA, UPS, INP; LISBP, F-31077 Toulouse, France

Full list of author information is available at the end of the article
}

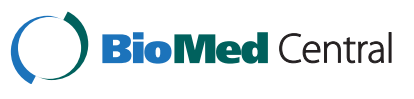

(C) 2015 Kempowsky-Hamon et al.; licensee BioMed Central. This is an Open Access article distributed under the terms of the Creative Commons Attribution License (http://creativecommons.org/licenses/by/2.0), which permits unrestricted use, distribution, and reproduction in any medium, provided the original work is properly credited. The Creative Commons Public Domain Dedication waiver (http://creativecommons.org/publicdomain/zero/1.0/) applies to the data made available in this article, unless otherwise stated. 


\section{Background}

Breast cancer, the most common invasive cancer in women, is an heterogeneous and complex disease. Currently, the management of breast cancer patients is based on clinicopathological characteristics such as age, menopausal status, tumor size, lymph node status, histologic grade [1] and on three immunohistochemical predictive markers: estrogen (ER) and progesterone (PR) receptors and human epidermal growth factor receptor 2 (HER2) [2,3].

Clinicopathological parameters and immunohistochemical markers are combined in guidelines such as St Gallen's consensus and Nottingham [4] prognostic Index or incorporated in internet algorithms such as Adjuvant!Online (https://www.adjuvantonline.com/index.jsp), for treatment decision making. The combination of these parameters provides assessments of the benefit of a systemic endocrine or chemo-therapy [5]. Although effective in the reduction of mortality, these principles have shown limits and are not sufficient for individualized medicine. Tumors with similar clinical characteristics can have noticeably different outcomes in terms of treatment response and survival. Furthermore, over-treatment with adjuvant therapy is not harmless [6].

For the past decade, genome-wide microarray-based expression profiling studies have been used as a powerful tool to improve understanding of the biology of breast cancer [7-12]. With these technologies, many prognostic gene expression signatures were identified to predict breast cancer recurrence risk [13-21]. Molecular differences even among tumors with similar pathological features have been unravelled and a new molecular taxonomy for breast cancer classification into several subgroups (luminal A and B, basal-like, HER2, normal breast-like) has been proposed. However, most of these gene signatures are still under development for prospective validation in clinical trials. Despite the promise of previous gene signatures, decisions making in clinical practice are still guided by traditional parameters. Integrating microarray information and using it as a complement to clinicopathological parameters could provide more accurate and robust prognostic tests in order to guide adjuvant systemic treatment that could reduce the cost of breast cancer treatment.

Biological data obtained from high throughput technologies (DNA microarray, NGS, and so forth) are known to generate certain level (amount) of imprecise and noisy data. Moreover, the high dimensionality of these technologies data (tens to hundreds of thousands of features) makes use of machine learning and data mining techniques necessary, since many of these features are irrelevant or redundant. Many research efforts have been directed in the last two decades towards developing efficient feature selection methods [22-25]. The existing methods are traditionally categorized as filter or wrapper methods, with respect to the criterion used to search for relevant features [26,27]. In filter approaches, features are scored and ranked according to a certain statistical criteria and those with the highest ranking values are selected. Most frequently used filter methods include $t$-test [28], chi-square [29], Wilcoxon [30], Pearson correlation coefficients [31] and Principal Component Analysis [32]. Filter methods are fast but lack in robustness against feature interactions and redundancy [33]. Besides, the way to determine the rankings cut-off point to select only truly important features and exclude noise is not clear. Wrapper methods use the performance of a learning method to assess the quality (accuracy) of the selected feature subset in predicting the target (e.g. determined by cross validation). Wrapper methods employ search algorithms to determine an optimal subset of features. The most generally used search approaches are backward elimination and forward selection [26]. Stochastic algorithms, developed for solving large scale combinatorial problems, such as ant colony optimization, genetic algorithms, and simulated annealing are also used [34-36]. Although these algorithms efficiently capture feature redundancy and interaction they are computationally expensive. Recently, some authors take advantage of both filter and wrapper methods and propose hybrid algorithms [33,37-39]. The idea is to apply first a filter method to select a feature pool and then the wrapper method is applied to determine the optimal subset of features from the selected pool. The most popular learning algorithm used in wrapper methods is the support vector machines (SVM) [40]. Nevertheless, the accuracy of an SVM depends on the choice of the parameters and the kernel function. SVMs are sensitive to noisy training data, which can degrade their performance. Even more, they are prone to over-fitting and poor generalization. The principal drawback of wrapper methods is the computational cost, since they evaluate the feature subset with a learning algorithm, which are usually iteratively. Therefore to enhance the wrapper approach performance it is necessary to use fast learning algorithms which performs well when dealing with noisy and imprecise data.

Fuzzy logic was introduced in 1965 by Zadeh [41], it deals with reasoning that is approximate rather than fixed and exact. Contrary to traditional Boolean logic, where objects are classified either true (1) or false (0), in fuzzy logic, they may have values ranking from 0 to 1 . Fuzzy logic has been widely used in system control due to its simplicity and effectiveness, especially in the case of nonlinear and highdimensional systems. This is mainly due to its fundamental concept which enables to handle and manipulate imprecise and noisy data. Additionally, it provides an intuitive interpretation of the results. Although some attempts to use fuzzy logic to perform feature selection have been proposed [42-49], certainly these methods perform well when dealing with imprecise and noisy data but they generally end up with a high sophistication. Either they depend on a specified method (e.g. Fuzzy C-Means [50]) 
which is designed originally for clustering, or they use an arbitrary choice to determine the linguistic terms of the "fuzzified" features, which is not always possible and accurate enough whenever a big number of features must be tackled. Moreover, in order to reduce computational cost, some authors have combined fuzzy selection mechanisms with genetic algorithms or even have introduced the concept of fuzzy entropy for selecting relevant features [44-46].

Fuzzy classifiers have recently shown their effectiveness in classification tasks, since they enable dealing with noisy and imprecise information which is often present in many applications. However, their performances decrease significantly in case of high dimensional and/or heterogeneous problems. Despite these drawbacks, an increasing interest in applying fuzzy classifiers to breast cancer prognosis, using gene expression data has been observed [51,52].

In contrast to previous studies where fuzzy logic was used for assessing patient classification, we herein developed feature selection and classification algorithms both based on the fuzzy logic concept of membership degree. Our approach involves feature weighting based on a membership margin in order to improve the performance of fuzzy classifiers on high dimensional and heterogeneous problems. The effectiveness of this method has been previously demonstrated on problems involving mixed type of data (numerical, qualitative, symbolic intervals) [53].

One of the most important parameter in breast cancer is the histological tumor grade which classifies patients in three classes: 1,2 or 3 . Histologic grade 1 and 3 statuses are related to a low and high risk of recurrence, respectively. However, about $50 \%$ of tumors are classified as histologic grade 2 , the moderately differentiated grade $[13,14]$. This grade is associated with an unclear risk of recurrence and is thus not informative for clinical decision making. Individualized medicine, not only based on clinicopathological characteristics, but also using information derived from tumor molecular profiles could improve patient management and increase survival. However it remains a clinical challenge. We have applied this new fuzzy logic methodology on gene expression data information obtained from public available datasets and our own patients cohort. We identified gene signatures enabling accurate discrimination of patients with breast cancer into either grade 1 or grade 3 and to help classifying patients with histologic grade 2 tumors into grade 1 or grade 3 like. This classification could lead to a reduction of over- and under- treated patients.

\section{Results}

Gene signatures according to fuzzy molecular grade ( $f M G$ ) We applied the fuzzy logic selection algorithm MEMBAS on four public available cohorts to identify prognostic gene expression signatures for breast cancer, based on histologic grade. The use of several cohorts allowed taking into account a maximum heterogeneity of patients. Due to the different microarray platforms (Affymetrix, Agilent Technologies), experimental protocols and data processing for normalization used by authors, we choose to use gene expression raw data individually. In the training sets, we analyzed expression profiles of 452 patients with primary breast cancer with histologic grade 1 or 3 . Histologic grade 2 patients were excluded since our objective was to identify low and high risk profiles in order to classify later intermediate grade tumors as molecular grade 1like (fMG1) or 3-like (fMG3). Tumor data were derived from four independent cohorts: NKI2-Agilent (113 samples) [16], KJX64KJ125-GSE2990 (103) [14], Uppsala-GSE4922 (123) [13], and Transbig-GSE7390 (113) [6]. The training sets were used to identify groups of genes whose expression allowed distinguishing histologic grade 1 from grade 3 tumors. MEMBAS algorithm ranked in decreasing order the probes and a posterior probability was iteratively estimated for each tumor by leave-one-out cross validation (LOOCV). We performed selection of the minimal number of probes which achieved the highest possible sensitivity (Histologic Grade HG3 patients correctly identified) and an acceptable level of specificity (HG1 patients correctly classified), in order to have accurate grade assignments (Figure 1). In breast cancer context, a high sensitivity is privileged over high specificity in order to be sure that patients with high risk of recurrence would be thoroughly treated.

We identified 67 Agilent probes and 38 Affymetrix probes respectively for the gene signatures $A$ and $B$, designed from only ER positive patients of NKI2 and KJX64-KJ125 cohorts; whereas gene signatures C and D were composed of respectively, 71 and 18 Affymetrix probes selected on both ER positive and negative tumors for Uppsala and Transbig cohorts. These probe sets correspond respectively to $65,37,65$ and 16 unique genes respectively. Most genes were overexpressed in grade 3 tumors. As highlighted by molecular taxonomy, ER positive and ER negative breast cancers are fundamentally different diseases and ER status may influence gene expressions. For this reason, we tested ahead stratification of tumors, according to their ER status (either only ER positive or both ER positive and negative) and selected the gene signatures with the best discriminant power for each cohort, regardless of ER status for patients included in the training set.

When we compared the new gene signatures obtained with our fuzzy logic strategy with the previously published prognostic signatures, we found several genes in common (Additional file 1: Table S1). For example in gene signature C [13], 14 of the 18 genes from the PAM genetic grade signature [13] were identified. More than 

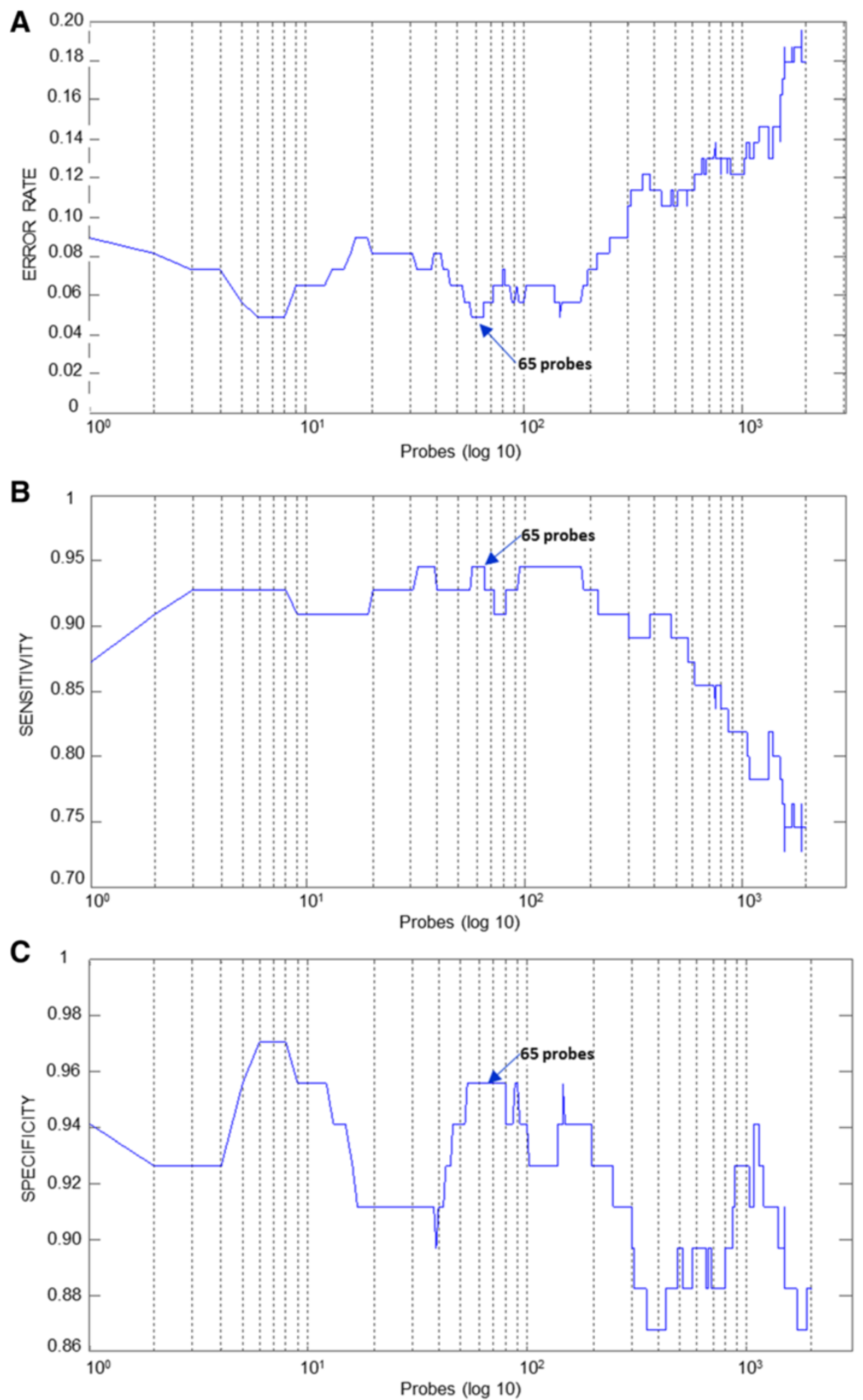

Figure 1 Selection of the most discriminant probes for GS C - GSE4922 [13]. Minimum number of probes providing the best sensitivity with a low global error. A) Global Error, B) Sensitivity, C) Specificity. The number of evaluated probes is expressed in a log 10 scale (horizontal axis).

$77 \%$ of the genes selected in Affymetrix gene signatures B [14], C [13] and D [6] were common to Sotiriou's GGI signature [14].

\section{Grade $1 \& 3$ profiles - training set}

We examined the performance of our gene signatures in predicting histologic grade 1 and 3 by determining whether 
the classification obtained by this molecular method agrees with the histologic ones. Sensitivity and specificity rates of each signature on the respective training sets are summarized in Table 1 . Best results were obtained with gene signature $C$ on Uppsala cohort [13]. Only 3 out of 55 histologic grade 3 tumors (5\%) were classified in molecular grade 1-like and 5 out of 68 histologic grade 1 tumors (7\%) were assigned to molecular grade 3-like. Secondly, gene signatures A [16] and B [14] achieved a sensitivity of $90 \%$ (5/49 and 4/40 misclassifications of grade 3 ) and a specificity of $86 \%$ and $87 \%$ respectively (9/64 and 8/63 misclassifications of grade 1). Gene signature D [6] presented a high rate of sensitivity (93\%) and a lower specificity (67\%), likely due to an imbalance in the composition of grade 1 and 3 tumors in this cohort and to a lower number of grade 1 tumors. To sum up, cancer grades prediction obtained from the four signatures strongly correlated with those obtained from histologic data.

As detailed in the Methods section (Figure 2), to quantify the degree of membership to molecular grade 1-like (fMG1) and 3-like (fMG3), a molecular grade score was developed. As shown in Figure 2, a tumor displaying a grade 3-like gene expression profile is assigned with a score (membership degree) greater than or equal to 0.5 , whereas a score below 0.5 corresponded to a tumor displaying a grade 1-like gene expression profile. The values of molecular grade score for all datasets are shown in Figure 2.

Molecular grade scores were well correlated to the gene expression patterns in Figure 3. The four gene signatures with molecular grade scores accurately classified grade 1 and 3 tumors with few misclassifications.

\section{Grade 2 classification - training set}

To determine if we were able to separate histologic grade 2 tumors in two classes, i.e. grade 1-like and grade 3-like, we used the same classification algorithm (i.e. LAMDA)

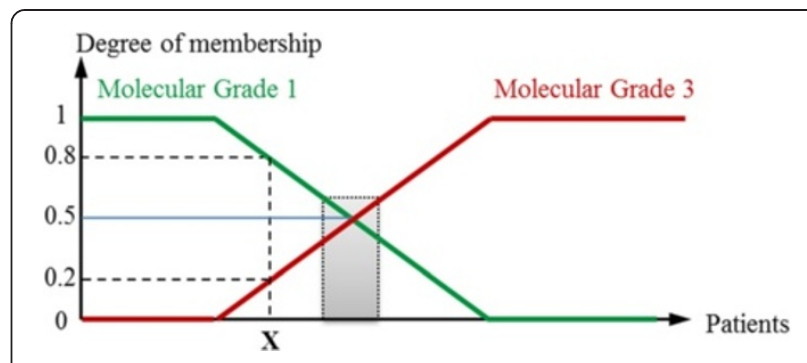

Figure 2 Membership degree of a patient $X$ to both molecular grade $\mathbf{1}$ (green) and grade $\mathbf{3}$ (red) classes. The gray rectangle, for membership values around 0.5 , corresponds to an uncertainty zone where gene expression values are intermediate.

preserving the same profiles of histologic grade 1 and 3 tumors (Table 1). The gene expression profiles of the histologic grade 2 tumors were similar to either molecular grade 1 profile or molecular grade 3 profile (Figure 3 ). Thus Figure 3 shows that most grade 2 tumors can be molecularly separated either into grade 1 -like or grade 3like classes.

Although our fuzzy gene signatures could accurately distinguished grade 1 from grade 3 tumors and separated grade 2 tumors in grade 1 like or grade 3 like, we defined an indecision zone that scored arbitrarily between 48 and $52 \%$, due to the equivocal gene expression grade profile of these patients (Figure 3 Heat map). For patients whose classification score lies within this uncertainty zone, their gene expression grade profile cannot be determined with certainty. Equivocal profiles represented a sizeable part of tumors (20 to $41 \%$ ) according to the cohort, and this is particularly highlighted by the slope of the grade 2 molecular score on the fGS C (Figure 3, fGS C bottom panel). The origins of these intermediate tumors are unclear and could be biological or technical.

Table 1 Classification agreement between molecular (fMG) and histologic grades (HG) in training cohorts

\begin{tabular}{|c|c|c|c|c|c|c|c|c|c|}
\hline \multirow{3}{*}{$\begin{array}{l}\text { Gene } \\
\text { signature }\end{array}$} & \multirow{3}{*}{$\begin{array}{l}\text { Training } \\
\text { cohort }\end{array}$} & \multirow{3}{*}{$\begin{array}{l}\text { Total } \\
\text { patiens (N) }\end{array}$} & \multirow{3}{*}{$\begin{array}{l}\text { Molecular } \\
\text { grade (fMG) }\end{array}$} & \multicolumn{6}{|c|}{ Histologic grade } \\
\hline & & & & \multicolumn{2}{|c|}{ HG1 } & \multicolumn{2}{|c|}{ HG3 } & \multicolumn{2}{|c|}{ HG2 } \\
\hline & & & & $\mathbf{n}$ & $\%$ & $\mathbf{n}$ & $\%$ & $n$ & $\%$ \\
\hline \multirow[t]{2}{*}{ fGS A } & NKI2 [16] & 206 & G1 & 55 & (86) & 5 & (10) & 46 & $(49)$ \\
\hline & & & G3 & 9 & (14) & 44 & $(90)$ & 47 & (51) \\
\hline \multirow[t]{2}{*}{ fGS B } & KJX64-KJ125 [14] & 166 & G1 & 55 & $(87)$ & 4 & $(10)$ & 38 & $(60)$ \\
\hline & & & G3 & 8 & (13) & 36 & $(90)$ & 25 & $(40)$ \\
\hline \multirow[t]{2}{*}{ fGS C } & Uppsala [13] & 249 & G1 & 63 & (93) & 3 & (5) & 82 & $(65)$ \\
\hline & & & G3 & 5 & (7) & 52 & (95) & 44 & (35) \\
\hline \multirow[t]{2}{*}{ fGS D } & TVBDX [6] & 196 & G1 & 20 & $(67)$ & 6 & (7) & 32 & (39) \\
\hline & & & G3 & 10 & (33) & 77 & (93) & 51 & $(61)$ \\
\hline
\end{tabular}

\% in bold correspond to specificity (HG1) and sensibility (HG3). 


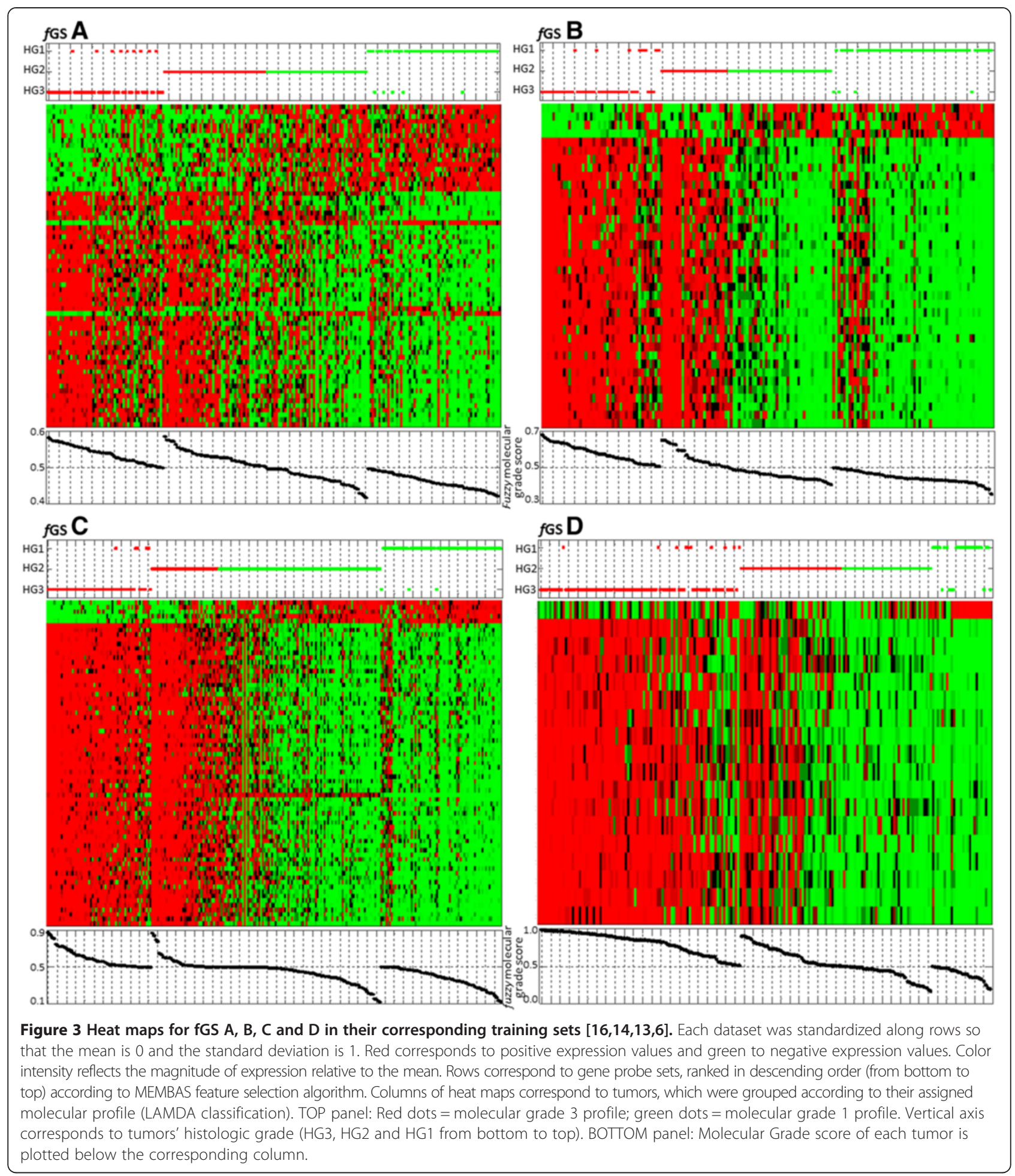

Gene signature (GS) performance assessment in independent validation sets

An important aspect of our fuzzy gene signatures (fGS) is their performance. It has to be assessed on cohorts from various origins, including a validation set. Therefore, we tested the four fuzzy gene signatures (fGS) on several independent patient cohorts. We used exactly the same probes than the ones included in each fGS in 
order to strictly respect selected molecular profiles. Only gene signature A could not be validated on an independent dataset due to the deficiency of other Agilent platform datasets. Furthermore, redundant patients present in KJX64/KJ125 and Uppsala datasets were removed from the validation tests so they were only considered once. Validation tests showed that gene signatures B, C and D achieved similar performances to those obtained in training sets with about $80-90 \%$ of sensitivity (classification of HG3 tumors) and specificity (classification of HG1 tumors), and error rates between 7 to $17 \%$ (Additional file 2: Table S2). Figure 4 shows the cross validation fGS $\mathrm{B}$ on the different cohorts. We observed a suitable agreement in classification between molecular

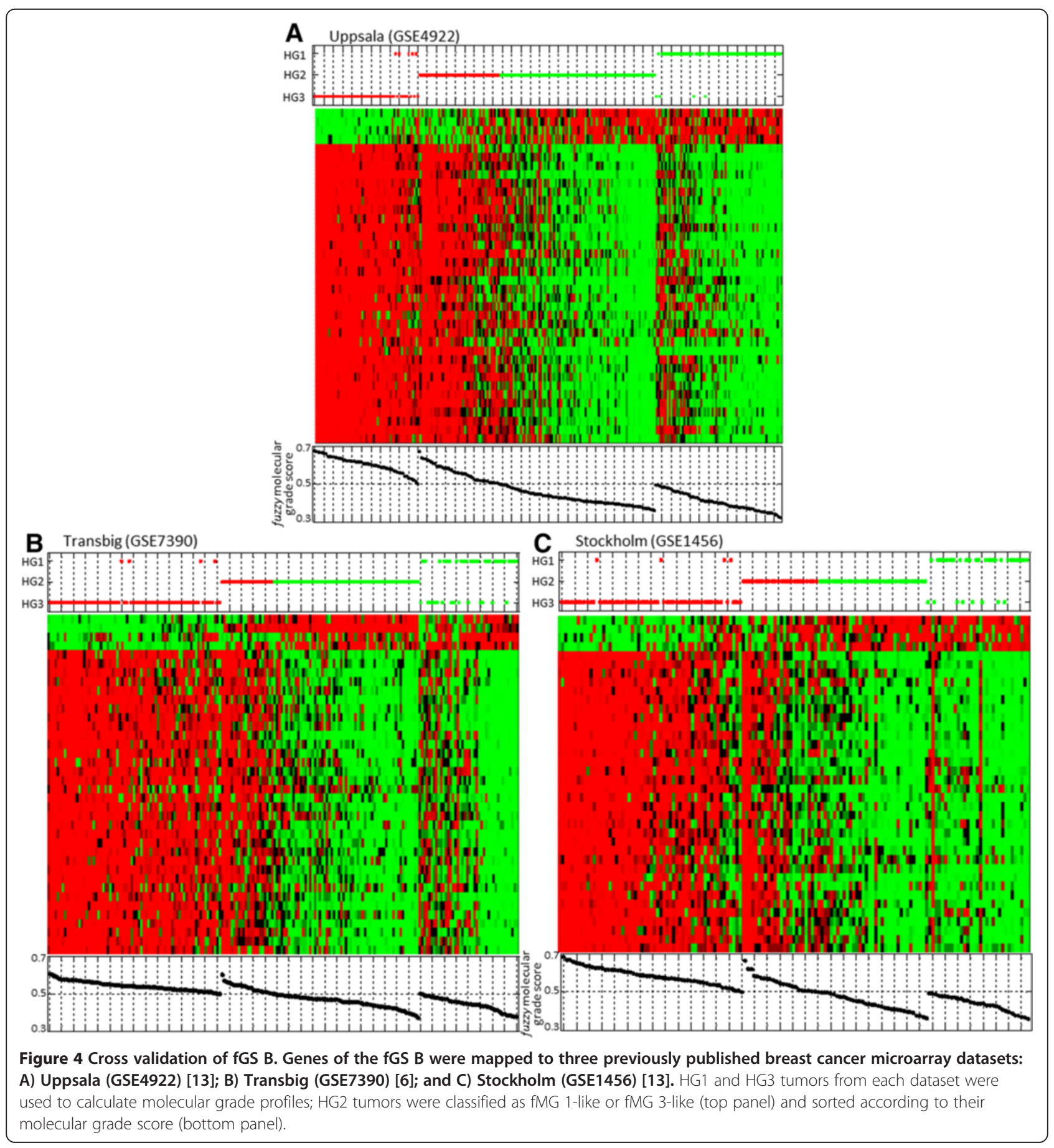


and histologic grades. The gene expression profiles of histologic grade 1 and 3 tumors for each gene signature were similar to those identified in the training set (Figure 3 $f G S \mathrm{~B})$. We also found, as in the training set, close associations between the gene expression profiles, fuzzy molecular grade scores and histologic grades. We can notice that it exhibited good results with both ER positive and negative tumors although its fuzzy molecular grade score was constructed initially with only ER positive tumors to avoid bias. Furthermore, we calculated fuzzy molecular grade scores for grade 2 tumors in validation sets. We observed that, albeit an equivocal zone was observed, most grade 2 tumors could be separated in fMG 1-like and fMG 3-like (Figure 4).

\section{Correlation between fuzzy molecular grade score and relapse-free survival}

To estimate the prognostic performance of gene signatures in breast cancer patients, the Kaplan-Meier method [54] was used to compare the relapse-free survival deduced from histologic classification to that from fuzzy molecular classification.

The reliability of molecular grades in the different datasets and in pooled datasets was tested. Histologic grade 3 tumors were associated with a high rate of relapse whereas histologic grade 1 tumors were related to a low risk of recurrence. Histologic grade 2 tumors were associated with an intermediate rate of relapse. We performed the same survival analysis with fuzzy molecular grade obtained with the four gene signatures. The results summarized by hazard ratios, 95\% confidence intervals and $\mathrm{p}$ value, are presented in Table 2. The hazard ratio from all datasets classified with the four gene signatures showed significant log rank test $\mathrm{p}$ value. Only gene signature $\mathrm{D}$ failed to reach significance in its training set (Transbig cohort), however histologic grading did too. As shown in Table 2, discriminating grade 1 from grade 3 tumors with our Fuzzy molecular method exhibited better results than with the histologic one. In addition, while survival curves of molecular grade 1 and 3 were similar to those of histologic grade 1 and 3 respectively, in some cases, fuzzy gene signatures could be better than the histologic grade classification in improving relapse free survival for patients with grade 1 and 3 tumors (Additional file 3: Figure S1).

To evaluate the clinical interest of molecular grades, grade 1-like and grade 3-like, we compared the relapsefree survival of grade 2 patients who had grade 1-like profile with that of patients who had grade 3-like profile, according to the four gene signatures. The four fuzzy molecular grade signatures separated grade 2 tumors into 2 distinguished classes, grade 1-like and grade 3 -like, with a statistically significant difference in relapse-free survival across all datasets or when

Table 2 Survival analysis of grade 1 and 3 tumors classified with fuzzy molecular and histologic grades

\begin{tabular}{|c|c|c|c|c|c|}
\hline \multirow{2}{*}{$\begin{array}{l}\text { fuzzy Gene } \\
\text { Signature }\end{array}$} & \multirow{2}{*}{$\begin{array}{l}\text { HG } 1 \& 3 \text { patients } \\
\mathrm{n}\end{array}$} & \multicolumn{2}{|l|}{ fuzzy Gene Signatures } & \multicolumn{2}{|l|}{ Histologic grade } \\
\hline & & Hazard Ratio $(95 \% \mathrm{Cl})$ & $\mathrm{p}$ value logrank test & Hazard Ratio $(95 \% \mathrm{Cl})$ & $\mathrm{p}$ value logrank test \\
\hline \multicolumn{6}{|l|}{$f M G A$} \\
\hline $\mathrm{NKI} 2$ training & 113 & $2.136(1.466-3.113)$ & $<.001$ & $1.5896(1.135-2.213)$ & $<0.0052$ \\
\hline NKI2 validation all & 163 & $1.989(1.463-2.704)$ & $<.001$ & $1.694(1.294-2.218)$ & $<.001$ \\
\hline \multicolumn{6}{|l|}{ fMG B } \\
\hline KJX64/KJ125 training & 99 & $1.923(1.342-2.754)$ & $<.001$ & $1.546(1.075-2.223)$ & $<0.0184$ \\
\hline Transbig & 113 & $1.435(1.005-2.051)$ & $<0.0426$ & $1.062(0.792-1.426)$ & $=0.0541$ \\
\hline Stockholm & 89 & $2.711(1.313-5.595)$ & $<0.00158$ & $2.104(1.28-3.459)$ & $<0.0103$ \\
\hline Pool 1 & 272 & 1.59 (1.25 to 2,02$)$ & $<0.0001$ & 1.55 (1.20 to 2.00$)$ & $<0.001$ \\
\hline \multicolumn{6}{|l|}{$f M G C$} \\
\hline Uppsala training & 123 & $1.484(1.091-2.017)$ & $<0.0103$ & $1.773(1.306-2.408)$ & $<.001$ \\
\hline Stockholm & 89 & $4.134(1.518-11.258)$ & $<.001$ & $2.104(1.28-3.459)$ & $<0.0103$ \\
\hline \multicolumn{6}{|l|}{$f M G D$} \\
\hline Transbig Training & 113 & $1.28(0.852-1.923)$ & $=0.23$ & $1.062(0.792-1.426)$ & $=0.0541$ \\
\hline KJX64/KJ125 & 122 & $1.591(1.157-2.186)$ & $<0.00312$ & $1.518(1.11-2.077)$ & $<0.00725$ \\
\hline Stockholm & 89 & $3.844(1.412-10.469)$ & $<.001$ & $2.104(1.28-3.459)$ & $<0.0103$ \\
\hline Pool 2 & 281 & 1.65 (1.31 to 2,07$)$ supp KJX & $<0.00001$ & 1.66 (1.32 to 2.09$)$ & $<0.00001$ \\
\hline
\end{tabular}

Pool 1: Uppsala + Transbig + Stockholm.

Pool 2: KJX64/KJ125 + Uppsala + Stockholm. 
datasets were pooled (Figure 5 and Additional file 4: Table S3). Grade 2 tumors with grade 1-like profile had a lower risk of recurrence than grade 3 -like.

We examined several variables in a univariate analysis (Additional file 5: Table S4) and found that gene expression grade of our fGS, histologic grade, lymphoma node status and tumor size were all statistically significantly associated with relapse-free survival. However, in a multivariable analysis, only our fuzzy Gene Signature (fGS) and tumor size remained statistically significant associated with relapse-free survival.
Our fGS having the strongest association: $\mathrm{HR}=1.51$, $95 \% \mathrm{CI}=1.21$ to $1.88 ; \mathrm{P}<0.0004$.

\section{Fuzzy molecular grade ( $\mathrm{FMG}$ ) validation on an} independent cohort

\section{ICR cohort on Nimblegen custom microarray}

In order to evaluate the performance of our four gene signatures, we designed a NimbleGen custom microarray with the genes obtained on our gene signatures and validated them thanks to expression profiles of breast cancer tumors from a new cohort (C. Regaud Institute,

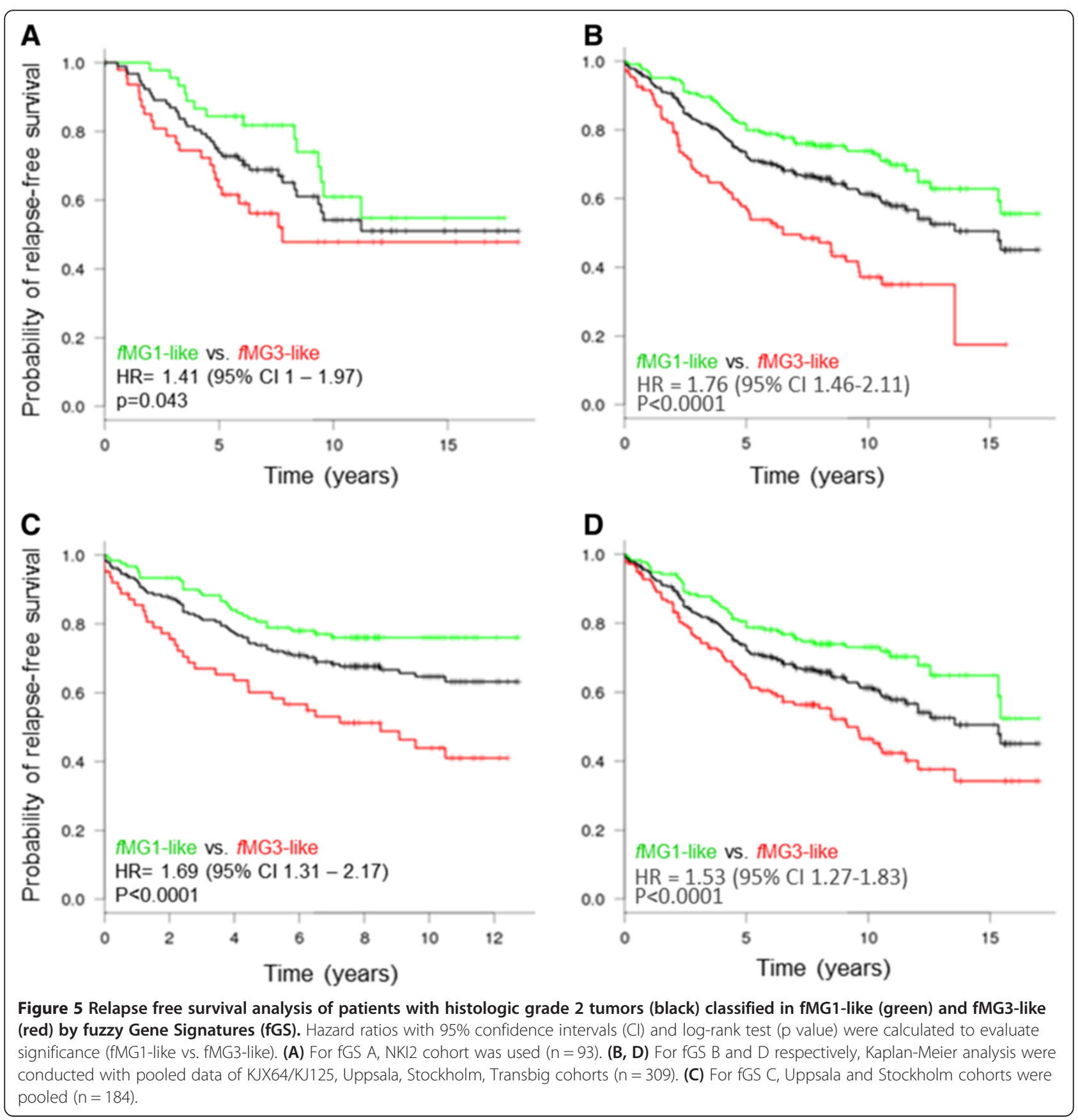


$\mathrm{n}=150)$. This cohort consists of 18 histologic grade 1 tumors (11.9\%), 58 (38.7\%) grade 2 and 74 (49.3\%) grade 3 tumors. The custom NimbleGen array was composed of genes of interest with 9 probes for each transcript. We used MEMBAS selection algorithm, instead of the conventional mean value, in order to rank (descending order) all probes representing the genes included in a gene signature. We selected the best ranked probe for each gene (Additional file 5: Table S4). Fuzzy molecular grade 1 and 3 profiles were determined with these selections and with LAMDA classification algorithm.

\section{Grade $1 \& 3$ profiles}

With Nimblegen microarrays constructed with the newly designed probes based on our fuzzy gene selection as

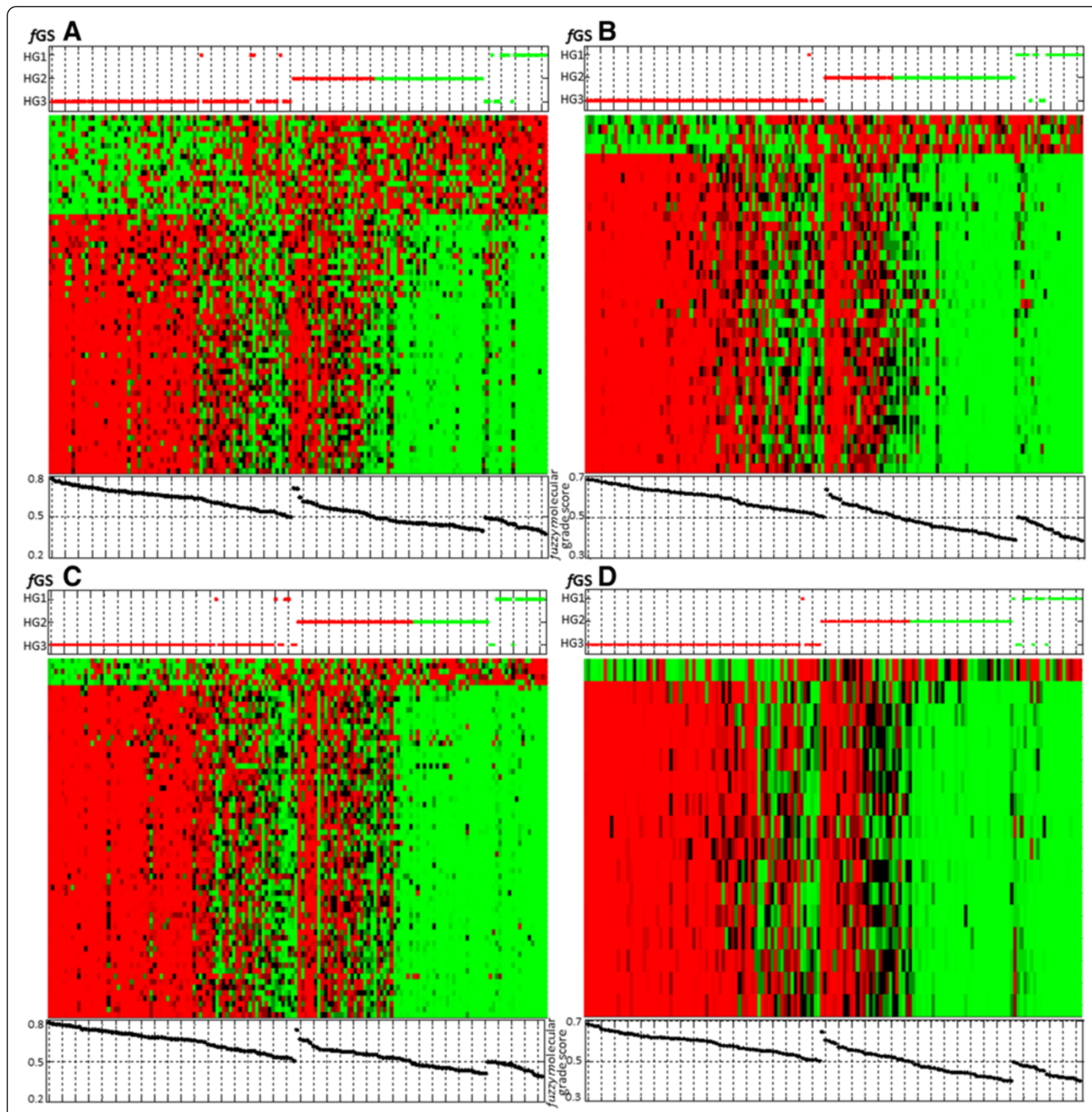

Figure 6 Heat maps for fuzzy gene signatures A, B, C and D in the validation set (ICR): for each fGS, HG1 and HG3 tumors were used to calculate molecular grade profiles; HG2 tumors were classified as fMG 1-like or fMG 3-like (top panel) and sorted according to their fuzzy molecular grade 3 score (bottom panel). 
described in Material and methods, we examined the expression profiles on ICRs cohorts for consistency with predicted histologic grade. As shown in Figure 6, the gene expression patterns of patients with histologic grade $1(n=18)$ and grade $3(n=74)$ tumors were similar to those identified previously in the training and validation sets from public datasets.

A total of 125 probe sets (representing 122 genes) were identified as genes with the highest discriminating power (i.e. the most significantly differentially expressed genes) between grade 1 and grade 3 tumors.

For all fuzzy gene signatures, we could easily discriminate low and high grade from the gene expression patterns of the ICR cohort. The accuracy of fuzzy molecular grading in terms of classified in low and high grade (fMG1 and fMG3) was evaluated using a LOOCV, since the number of grade 1 tumors is very small (11.9\%) compared to the number of grade 3 tumors (49.3\%). Results for high sensitivity and specificity are shown in Additional file 6: Table S5. For example, using fGS B, only one of the 18 HG 1 tumors (6\%) showed a fuzzy molecular grade score greater than 0.5 , and only three of the 74 HG 3 tumors $(4 \%)$ displayed a fuzzy molecular grade score inferior to 0.5 .

Most importantly, we noticed that misclassifications between gene signatures often corresponded to the same tumors. Indeed, in all four gene signatures, four tumors consistently showed an opposite molecular grade as compared to their histologic grade. Other tumor misclassifications were obtained in two or only one gene signature. Several of these tumors presented clinicopathological features that could explain these problems of concordance. Among the misclassified histologic grade 3 tumors, two tumors were invasive lobular carcinoma, of pleomorphic subtype (cases \#37 and 113). One tumor corresponded to a heterogeneous tumor (as of micropapillary and IDC-NST histologic subtypes) (case \#109). Another tumor displayed a triple negative phenotype (case \#127). Two misclassified histologic grade 1 tumors were heterogeneous tumors with distinct components (cases \#6 and 96), easily identified by microscopic examination (one case harboring a mucinous component, the one measuring $23 \mathrm{~mm}$ of greater size).

Grade 2 classification in $f M G 1$-like or $f M G 3$-like categories After checking the accuracy of the fuzzy prediction model on grade 1 and 3 tumors with the new design of probes, we tested the profiles of histologic grade 2 tumors $(n=58)$. As for the public datasets, the histologic grade 2 tumors harbored extreme values than encompassed those of histologic grade 1 and 3 tumors (Figure 6). It could be noticed that $69 \%$ of grade 2 tumors were classified identically by the four gene signatures. Only 7\% (4/58 tumors) of grade 2 tumors were ambiguously classified as fMG1-like by two gene signatures and as fMG3-like by the two others. Among these 4 tumors, 3 were within the equivocal zone. This demonstrates that the use of several gene signatures may prove useful to enhance confidence on prognostic information provided by molecular grade.

\section{Discussion}

It has been shown that breast cancer histologic grade provides an important prognosis information, with grade 1 tumors showing a low risk of recurrence as opposed to grade 3 tumors harboring a high risk of recurrence. However, about $50 \%$ of breast cancers are classified as moderately differentiated (intermediate) histologic grade 2 , which gives no information about the clinical decision or the treatment strategy to apply. Microarray gene expression data has been employed in order to increase the prognostic value of tumor grading, on the one hand by refining grade 2 tumors into two distinct categories which could be eventually related to low and high risk, and on the other hand improving reproducibility. In contrast to what has already been done, we have herein used a feature selection algorithm and a classifier based on fuzzy logic theory and concepts, in order to identify genes that best discriminate grade 1 from grade 3 tumors. We applied this approach to four previously published microarray datasets (Agilent and Affymetrix technologies) and generated four distinct fuzzy gene signatures. We found that all four grade signatures were able to reliably identify histologic grade 1 and 3 tumors and molecularly separate grade 2 tumors into fMG1-like or fMG3-like categories, regardless of the platform technology, experimental protocols or type of cohorts. The fact of applying the membership concept (i.e. a sample belongs simultaneously to each category with a given value of confidence) allowed representing the heterogeneity of tumors without the necessity of preprocessing into molecular subtypes. Hence, only those probes that truly contributed to the characterization of both grade 1 and grade 3 categories were selected. Moreover, the proposed fuzzy gene signatures succeeded to extend good classification results in other populations. The results were reproducible and gene signatures were validated across cohorts.

\section{Identified genes from Fuzzy GSs}

As expected, the majority of genes selected with fuzzy logic encompassed genes involved in cell cycle control and proliferation. Only one gene, CENPA, was common to all four GS. The centromere protein A (CENP-A) is an essential centromere protein, required for chromosome segregation during cell division. CENP-A has been associated with high grade cancers and is a strong prognostic marker for distant relapse in ER-positive breast cancer. McGovern SL and their collaborators 
[55] demonstrated a clear relationship between the degree of expression of this essential protein and outcome in ER-positive breast cancer. Seven genes - AURKA, CDCA8, DDX39A, FOXM1, KIF2C, MELK, MKI67were common to GS B, C and D (Affymetrix technology) (see Additional file 7: Table S6 and Venn Diagram Additional file 8: Figure S2). From the 122 selected genes, 46 of them are unique to GS A (Agilent), and 10, 23 and 1 are unique to GS B, C and D respectively. For GS A and B, generated with ER positive samples only, 7 genes overlapped (CENPA, BIRC5, CCNB1, KIF20A, KPNA2, RACGAP1).

Comparison of our four GS (122 selected genes) with previously published genetic grade signatures revealed a significant degree of overlap, in particular with PAM (Ivshina et al.) and GGI (Sotiriou et al.), where for some GS more than 77\% of the genes overlapped. These findings not only confirm the good performance of our approach (in terms of biological coherence) but also reinforce the fact that gene expression-based profiles of histologic grade can contribute to patient prognosis identification.

\section{Equivocal cases}

Although gene signatures accurately classify grade 1 and 3 tumors and separate grade 2 tumors in grade 1-like or grade 3 -like, we noticed an uncertainty zone where molecular profiles were intermediate. This uncertainty zone (see Methods Figure 2) was confirmed by the molecular grade score value. In fact, for tumors displaying a score around 0.5 , their molecular profile did not show a clear similarity neither to grade 3 nor to grade 1 profiles. Whilst it has been recently advocated, no consensual definition of this uncertainty zone is currently available in the literature. Arbitrarily, we defined as equivocal those cases with a classification score between 0.48 and 0.52 . For patients whose classification score lies within this uncertainty zone, their gene expression grade profile cannot be distinctly determined as grade 1-like or grade 3-like.

When cross validation of our fuzzy gene signatures was performed on public cohorts, equivocal profiles (all histologic grades included) represented a more or less significant part of tumors, ranging from 12 to $31.3 \%$ depending on the cohort. Albeit the origins of these intermediate tumors remain unclear and debatable, it most likely corresponds to a true biological process, reflecting the spectrum and continuum of disease found in ER+ breast cancer, especially with regards to proliferation. Some of these equivocal cases could also represent heterogeneous tumors with a mixture of grade 1 cells and grade 3 cells.

Likewise, concerning the results obtained when applying our gene signatures to the ICR cohort we noted that gene signatures could disagree for some tumors in the assignment of histologic grade 2 into grade 1-like or grade 3-like. Thus, we investigated whether problematic histologic grade 2 tumors had extreme or intermediate profiles. We analyzed the range of scores of grade 2 tumors and we observed a continuous score slope as for grade 1 and 3 tumors. Furthermore, we noticed that gene signatures produced about 9\% of equivocal profiles (between 7 to $11 \%$ of the cases depending on the gene signature). Whatever the grade gene signature, an intermediate zone thus exists. Most of grade 2 tumors classified differently by several gene signatures also corresponded to equivocal cases (their classification score ranging between 0.48 and 0.52 ).

\section{Conclusion}

In conclusion, our approach of fuzzy genetic molecular grading allows refinement of histologic grade 2 tumors into fMG1-like and fMG3-like categories, although an uncertainty zone still remains. This suggests that genetic grade could be used, at some degree and at the very best in combination to clinicopathological parameters, to further improve outcome prediction of patients that should be spared of systemic adjuvant therapy. It seems that one unique Gene Signature might not be sufficient enough for decision making. Several gene signatures might thus be used to enhance confidence on prognosis provided by molecular grade. Moreover, the use of both clinicopathological characteristics and information derived from tumor molecular profiles could improve patient management and increase survival. It is noteworthy that our fuzzy feature selection algorithm and classifier is capable of treating features of different types simultaneously. The next challenge will be to include relevant clinicopathological characteristics into our fuzzy molecular grade signatures. Future works will be therefore oriented in evaluating our fGS performance in association to other parameters, in independent and larger cohorts.

Finally, we have demonstrated here the proof of concept of using fuzzy logic to select relevant biomarkers and to better evaluate risk. Individualized medicine remains a clinical challenge and will still need new prognostic and predictive biomarkers. The fuzzy logic method might prove of useful value in discovering new biomarker combinations in other main applications such as prediction of treatment response.

\section{Methods}

\section{Breast microarray datasets}

We collected four public available datasets from patients with primary breast cancer profiled using Affymetrix or Agilent DNA microarrays: the Netherlands Cancer Institute NKI2 dataset from van de Vijver et al. (256 patients), the KJX64/KJ125 datasets from Sotiriou et al. (189 patients), Uppsala dataset from Ivshina et al. 
(249 patients) and the Bordet Institute TRANSBIG dataset from Desmedt et al. (198 patients). Some samples of the NKI2 cohorts were excluded from our study due to missing or biased data. Redundant patients (74 samples) present in KJX64/KJ125 and Uppsala datasets were removed from the validation tests so they were only considered once. Gene expression and clinical data of public series were retrieved from Gene Expression Omnibus (GEO) public database http://www.ncbi.nlm.nih. gov/geo, author's website and publications $[6,13,14,16]$. All datasets were retrospective. They are described in Additional file 9: Table S7.

\section{Selection of grade gene signatures and class prediction}

To evaluate cancer recurrence risk, we identified gene signatures using information on the histologic grade. The aim was to classify grade 2 tumors with unknown outcome, into two subclasses: a grade 1-like subgroup with good outcome and a grade 3-like subgroup with poor outcome. For this, a feature selection technique and a classification algorithm based on fuzzy logic concepts were used.

\section{Fuzzy feature partition}

Fuzzy set theory was proposed by Zadeh in 1965 to mathematically model the imprecision inherent to some concepts [41]. In short, fuzzy sets theory allows an object to partially (simultaneously) belong to a set (class) with a certain degree of membership between 0 and 1 . In a machine learning framework, an approach is defined as "fuzzy" if we consider that an individual belongs to each class with a certain degree of membership, unlike the "crisp" ("hard") approaches where each individual is considered to belong only to one class [56]. Taking this in consideration, we can apply these concepts to the problem of classifying breast cancer patients according to their fuzzy molecular (gene expression) grade ( $\mathrm{AMG}$ ) profile corresponding to a grade 1-like profile (fMG1) and a grade 3-like profile (fMG3). Figure 2 shows a representation of the membership degrees associated to each class for a group of patients. Then, patient $\mathrm{X}$, in the figure, has a membership degree of 0.8 for $f \mathrm{MG} 1$ and 0.2 for $f \mathrm{MG} 3$. An ambiguous (or uncertainty) zone can be defined around a membership degree value of 0.5 , representing those patients for which a low or high risk profile cannot be clearly associated, i.e. their molecular grade profile is really intermediate (see "gray rectangle" on Figure 2).

\section{Grade-associated gene selection Fuzzifying gene expression data}

In order to represent all the features (gene expression values) of a sample (patient/tumor) by their memberships to a reference fuzzy partition (molecular grade 1-like \& 3like), we used membership functions denoted as $\mu_{k}^{i}$ based on the similarity (or distance) semantics [57]. As stated by Medasani and Kim [58], no measures are available to evaluate the goodness or correctness of a given membership function, nevertheless the success of an algorithm depends on the membership functions used. Several functions should be used in order to select the one that gives the best performance for pattern recognition according to the type of data (see Additional file 10). In this work, the fuzzy extension of the binomial function and the Gaussian function were used [58]. The first one, works extremely well when the observations are grouped, after standardization (see eq.1), around 0 or 1 , but may present instability or definition problems when data is concentrated around 0.5 . The second one is commonly used when the volume of the observed data is important, since it is very likely to follow a Gaussian or semi-Gaussian distribution. Also, this function measures the proximity to an estimated center. In this way gene expression values are transformed into the membership space without any information loss and are ready to be used for both feature selection and classification.

\section{Fuzzy feature selection algorithm - MEMBAS}

As previously mentioned existing feature selection algorithms are traditionally characterized as wrappers or filters according to the criterion used to search the relevant features $[27,28]$. We have recently proposed a new feature selection algorithm, referred to as MEMBAS for MEmbership Margin Based Attribute Selection [59] which enables to process similarly the three data types (numerical, qualitative, interval) based on an appropriate mapping using fuzzy logic concepts. The algorithm measures simultaneously the contribution of each gene for each of the two classes (in our case, Molecular Grade1 \& Grade3 tumors), in order to find the best discrimination. That is, it extracts the most pertinent markers since it is based on feature weighting according to the maximization of a membership margin. To avoid the heuristic search during the feature selection procedure, MEMBAS optimizes a membership margin based objective function by using classical optimization techniques providing an analytical solution [60].

\section{Class prediction - Fuzzy classification algorithm}

The learning and classification algorithm, LAMDA (Learning Algorithm for Multivariable Data Analysis) [61] has been used to generate the fuzzy partition that best discriminates histologic grade 1 and 3 patients according to their gene expression profiles, as well as to determine the probes that best fit this partition. LAMDA is a fuzzy methodology of conceptual clustering and classification which is based on finding the global membership degree of a sample to an existing class, considering all the contributions of each feature. This contribution is called the marginal adequacy 
degree (MAD). The MADs are calculated by means of a membership function and they are then combined using "fuzzy mixed connectives" as aggregation operators in order to obtain the global adequacy degree (GAD) of an element to a class. Finally a sample (tumor/patient) will be assigned to the class for which its GAD is the highest [62]. In Hedjazi et al. [60] and his PhD manuscript [63] an extensive experimental study, including a comparison with known feature selection methods has been performed on several datasets presenting mixed-type and high-dimensional data. The experimental results in these works show that MEMBAS leads to a significant improvement of classification performance of LAMDA (fuzzy classifier) as well as other well-known classifiers (k-NN, SVM). Moreover, the combined fuzzy model MEMBAS/LAMDA works well in datasets with mixedtype data, since the same fuzzifying process (membership functions) is used for both feature selection and classification. This provides a similar processing for each feature type with minimal loss of information.

\section{Fuzzy molecular grade - gene signature strategy}

For each breast cancer cohort we identified the most relevant genes using MEMBAS/LAMDA algorithms. The procedure was as follows:

1. The cohort database was separated into a training set (histologic grade 1 and grade 3 patients) and a validation set (histologic grade 2 and unknown grade patients). Gene expression values were then standardized within the interval [x_imin, $x_{-}$imax]. This linear re-scaling of the variable into the interval $[0,1]$ was performed according to equation (1) where $\hat{\mathrm{x}}_{i}$ is the $\mathrm{i}$-th gene probe (feature) and $x_{i}$ is its normalized value; $x \_$imin and $x \_$imax $x$ are the bounds of the $\mathrm{i}$-th probe for the corresponding cohort.

$x_{i}=\frac{\hat{x}_{i}-\hat{x}_{i \min }}{\hat{x}_{i \max }-\hat{x}_{i \min }}$

This standardization, based only on histologic grade 1 and grade 3 status, was necessary since gene expression values in different platforms are scaled by unknown parameters and because grade compositions diverge between datasets.

2. Next, each gene probe value was fuzzified using the training data based on the appropriate learning process (LAMDA). The resulting fuzzy sets represent the probe's (feature) membership to each of the two existing classes. Probe fuzzification was performed according to two membership functions proposed by Aguado and Aguilar in [64]. Both functions were tested in order to identify which gene subsets provided the highest discrimination power (the objective is to find the highest classification accuracy with a minimum number of probes).

3. Once all gene expression values from probes had been fuzzified, the fuzzy feature selection algorithm MEMBAS was performed to rank probes in descending order according to their resulting fuzzy weight $\left(w_{f}\right)$. Then, iteratively, the classification performance in terms of overall classification error, sensitivity (percentage of histologic grade 3 tumors correctly classified), and specificity (percentage of histologic grade 1 tumors correctly classified) was calculated using LAMDA classification algorithm. Hence, for each iteration, the number of probes was incremented, following the ranked order, until the whole probe set was tested. Since most cohort datasets are scarce and small (in terms of patient number), classification performance was evaluated with a leave-one-out cross-validation (LOOCV) to estimate the optimal classification parameters as proposed by Wessels et al. [65]. This method consists in holding out one single sample from the histologic grade 1 and grade 3 dataset of the cohort. This sample was considered as the validation data and the remaining histologic grade 1 and grade 3 samples of the cohort were used to estimate the optimal parameters of the classifier (profile of each class, exigency- $\alpha$ ). The resulting model was employed to classify the test sample. This was carried out on all samples so that each of them was used once for testing. This procedure is commonly used to evaluate the generalization performance of a classifier, since it reduces bias from the training set, i.e. data for a sample (tumor) are never used to estimate the classifier parameters (class profile) for its own classification. The resulting probe set, providing the higher classification performance, characterizes the fuzzy Molecular Grade 3-like (fMG3) and fuzzy Molecular Grade 1-like classes.

4. Given that LAMDA classification provides a global adequacy degree (GAD) for each sample to both classes (Figure 2), a molecular grade score has been introduced in order to determine the similarity between the Gene Expression Grade and the histologic grade. This score was determined by taking the GAD of each sample (tumor) for the Fuzzy Molecular Grade 3-like (fMG3) class. Then, if the score was greater than or equal 0.5 the patient is most likely to have a Grade 3-like gene expression profile (fMG3) and if the score was less than 0.5 the patient experiences a Grade 1-like gene expression profile (AMG1).

5. Once the optimal probe subset had been identified for a given cohort, the histologic grade 2 and unknown grade patients were classified into either fMG1-like or fMG3-like according to their resulting score (GAD). 


\section{NimbleGen Microarrays}

Gene signatures obtained were validated on an additional cohort. In order to focus the attention to the most informative genes, custom NimbleGen Roche $4 \times 72 \mathrm{~K}$ gene expression microarray kit (NimbleGen Roche Diagnostics, Meylan, France) was designed by NimbleGen support, based on genes included in gene signatures provided by the proposed fuzzy logic selection strategy (2030 accession numbers). We added controls of microarray experiment and housekeeping genes for normalization data. A mean of 9 probes by sequence were designed.

\section{Validation cohort}

In order to validate the different generated gene signatures (fGS), 150 frozen breast cancer tumors from the tumor bank of the Claudius Regaud Institute (ICR Toulouse, France) were selected. This cohort consisted of consecutive invasive breast carcinoma patients treated at Claudius Regaud Institute between 2009 and 2011. All patients included in this cohort signed an informed consent. Clinicopathological characteristics of the series were similar to those observed in routine clinical practice (i.e. majority of pre-menopausal patients presenting with T1c, node negative, ER+ invasive ductal carcinoma of intermediate grade). Clinical-pathological data of our samples are available in Additional file 11: Table S8. The study was approved by the local ethic committee.

\section{RNA extraction and microarray experiments}

Total RNA was extracted from $12 \mu \mathrm{m}$-thickcryostat sections of with Qiazol and RNeasy Lipid Tissue Kit (Qiagen, Courtaboeuf, France). The concentration of total RNA obtained from each tumor sample was determined using a Nanodrop spectrophotometer (Labtech, Palaiseau, France) and the integrity of the RNA was assessed using a 2100 Bioanalyzer $^{\circ}$ (Agilent Technologies, Massy, France). The percentage of tumor cells was evaluated on frozen a section stained with hematoxylin-eosin. Double-stranded cDNA (ds-cDNA) was synthesized from $2 \mu \mathrm{g}$ of total RNA using SuperScript One cycle kit (Invitrogen Life Technologies, Saint-Aubin, France) with random primers and Oligo(dT) primers, then cleaned with MinElute PCR Purification Kit (Qiagen, Courtaboeuf, France). ERCC RNA Spike-In Control Mix (Ambion Life technologies, Saint-Aubin, France), a set of external RNA positive controls, was added to total RNA at the beginning of the experiment to assess accuracy of measurements of gene expression. One $\mu \mathrm{g}$ ds-cDNA was Cy3 labeled using OneColor DNA labeling kit (NimbleGen Roche Diagnostics, Meylan, France). Hybridization on our Roche NimbleGen $4 \times 72 \mathrm{~K}$ custom microarray, washing slides and scan were performed according to the manufacturer's protocol (NimbleGen Roche Diagnostics, Meylan, France). DEVA software v1.2 (NimbleGen Roche Diagnostics, Meylan,
France) was used to extract the raw intensity values. Spike in control analyses, normalization and statistical analyses of the data were performed with Bioconductor packages (http://www.bioconductor.org/) and R software (version 2.14.1). All data were Robust Multichip Average (RMA) background corrected, log2-transformed, summarized by a robust mean for each probe. Then, housekeeping normalization was done on the experimental data. This method involved subtracting the average from a subset of 20 selected reference genes. As numerous studies reported that commonly used reference genes are not constantly expressed under different experimental conditions, we selected the 20 most suitable reference genes among 62 candidate genes previously reported as 'housekeeping' genes, based on their expression stability and correlation. Correlation coefficient between fluorescence and quantity of controls were comprised from 0.96 to 1 , providing high confidence in the experimental data. The microarray data have been deposited in the Gene Expression Omnibus (GEO) public database. The GSE53958 study can be found at: http://www.ncbi.nlm. nih.gov/geo/query/acc.cgi?acc=GSE53958.

\section{Survival analysis}

Disease-free survival was defined as the time interval from surgery until any type of recurrence (local, regional, or distant) or last date of follow-up [54]. Survival rates were estimated by the Kaplan-meier methods and comparison between groups were performed using Logrank test. Using cox proportional hazard modeling, hazard ratios were estimated with their corresponding 95\% confidence interval.

All P-values reported were two-sided. For all statistical tests, differences were considered significant at the $5 \%$ level. Statistical analyses were performed using $R$ software.

\section{Additional files}

Additional file 1: Table S1. Overlapped genes between GSs.

Additional file 2: Table S2. Validation tests; Agreement in classification between molecular and histologic grades in validation cohorts.

Additional file 3: Figure S1. Relapse free survival analysis of patients with histologic grade 1 (--_) and 3 (......) tumors classified in fMG1-like (green) and fMG3-like (red) by fuzzy Gene Signatures (fGS) in NKI2, KJX64/ KJ125, Transbig, Stockholm cohorts. Hazard ratios with 95\% confidence intervals $(\mathrm{Cl})$ and log-rank test ( $\mathrm{p}$ value) were calculated to evaluate significance (fMG1-like vs. fMG3-like and HG1 vs. HG3). (A) fGS $n^{\circ} A$. (B) fGS $n^{\circ}$ B. (C) fGS $n^{\circ} \mathrm{C}$. (D) fGS $n^{\circ} \mathrm{D}$.

Additional file 4: Table S3. Survival analysis of grade 2 tumors separated in grade 1-like and grade 3-like according to their molecular grade score.

Additional file 5: Table S4. Univariate and multivariate analysis of breast cancer prognostic factors for the fGS B $(n=118)$.

Additional file 6: Table S5. Agreement in classification between molecular and histologic grades in C. Regaud cohort. 
Additional file 7: Table S6. Selected NimbleGen' probes for the four gene signatures.

Additional file 8: Figure S2. Venn diagram showing the overlap between the four new gene signatures fGS A, fGS B, fGS C and fGS D obtained when we applied the fuzzy logic selection on breast cancer microarray databases.

Additional file 9: Table S7. Microarray datasets used for gene signature generation: distribution of patients according to clinical variables.

Additional file 10: Including mathematical detail of both feature selection (MEMBAS) and classification (LAMDA) algorithms.

Additional file 11: Table S8. Patients' characteristics from Claudius Regaud Institute.

\section{Competing interests}

The authors declare that they have no competing interests.

\section{Authors' contributions}

TKH(post-doctoral position) and CV(engineer) are the two people employed with ANR temporary contract on this project. They did all the fuzzy logic selection and Nimblegen microarrays experiments and interpretations (contributions to conception and design, acquisition of data, analysis and interpretation of data). LH student, participated in the fuzzy logic development. $L T, L P R$, LM engineer who did all the RNA breast cancer extraction and control quality. TF, DL and SL, engineer that performed the statistical analysis and deposited the data in the Gene Expression Omnibus (GEO) public database. FD and GF revised the manuscript critically for important intellectual content. JMF: INNODIAG ANR coordinator gave a final approval of the version to be published. MLT, MVLL and VAL are the 3 principal partners of the INNODIAG ANR project and conceived the study, and participated in its design and coordination and helped to draft the manuscript. All authors read and approved the final manuscript.

\section{Acknowledgements}

This work was supported by the National Research Agency (Agence National pour la Recherche), "BIOTECHNOLOGIES" program (decision n ANR 2010 BIOT 004 06: Project INNODIAG) and by the "pôle de compétitivité" Cancer-Bio-Santé from Midi Pyrénées, France. Carine Valle and Tatiana Kempowsky-Hamon were supported by ANR fellowship. We thank the ethics committee "Claudius Regaud Cancer Institute Research committee" that approved our study and gave us access to the biological resources and patient database. We also thank our colleagues at the GeT-Biochip Platform Toulouse Genopole and Dendris Company for fruitful discussion and critical reading of the manuscript.

\section{Author details}

'CNRS, LAAS, F-31400 Toulouse, France. ' ${ }^{2}$ niversité de Toulouse; INSA, UPS, INP; LISBP, F-31077 Toulouse, France. ${ }^{3}$ INRA, UMR792, Ingénierie des Systèmes Biologiques et des Procédés, F-31400 Toulouse, France. ${ }^{4}$ CNRS, UMR5504, F-31400 Toulouse, France. ${ }^{5}$ Institut Claudius Regaud, Biology and Pathology Department; INSERM UMR1037, Toulouse, France. ${ }^{6}$ Dendris SAS, 8 Rue de Cugnaux, 31300 Toulouse, France. ${ }^{7}$ Institut Claudius Regaud, Oncology Department, Toulouse, France.

\section{Received: 17 January 2014 Accepted: 12 January 2015}

\section{Published online: 07 February 2015}

\section{References}

1. Elston CW, Ellis IO. Pathological prognostic factors in breast cancer. I. The value of histologic grade in breast cancer: experience from a large study with long-term follow-up. Histopathology. 1991;19(5):403-10.

2. Sotiriou C, Pusztai L. Gene-expression signatures in breast cancer. N Engl J Med. 2009;360:790-800.

3. Colombo PE, Milanezi F, Weigelt B, Reis-Filho JS. Microarrays in the 2010s: the contribution of microarray-based gene expression profiling to breast cancer classification, prognostication and prediction. Breast Cancer Res. 2011;13:212.

4. Goldhirsch A, Winer EP, Coates AS, Gelber RD, Piccart-Gebhart M, Thürlimann B, et al. Personalizing the treatment of women with early breast cancer: highlights of the St Gallen International Expert Consensus on the Primary Therapy of Early Breast Cancer. Ann Oncol. 2013;2013(24):2206-23.

5. Fumagalli D, Andre F, Piccart-Gebhart MJ, Sotiriou C, Desmedt C. Molecular biology in breast cancer: should molecular classifiers be assessed by conventional tools or by gene expression arrays? Crit Rev Oncol Hematol. 2012;84:58-69.

6. Desmedt C, Piette F, Loi S, Wang Y, Lallemand F, Haibe-Kains B, et al. Strong time dependance of the 76- gene prognostic signature for node-negative brest cancer patients in the transbig multicenter independant validation series. Clin Cancer Res. 2007:13(11):3207-14.

7. Perou CM, Sorlie T, Eisen MB, van de Rijn M, SS I r, Rees CA, et al. Molecular portraitsof human breast tumours. Nature. 2000;406:747-52.

8. Hu Z, Fan C, Oh DS, Marron JS, He X, Qagish BF, et al. The molecular portraits of breast tumors are conserved acrossmicroarray platforms. BMC Genomics. 2006;7:96

9. Sorlie T, Tibshirani R, Parker J, Hastie T, Marron JS, Nobel A, et al. Repeated observation of breast tumor subtypes in independent gene expression datasets. Proc Natl Acad Sci U S A. 2003;100:8418-23.

10. Sorlie T, Perou CM, Tibshirani R, Aas T, Geisler S, Johnsen H, et al. Gene expression patterns of breast carcinomas distinguish tumor subclasses with clinical implications. Proc Natl Acad Sci U S A. 2001:98:10869-74.

11. Desmedt C, Haibe-Kains B, Wirapati P, Buyse M, Larsimont D, Bontempi G, et al. Biological processes associated withbreast cancer clinical outcome depend on the molecular subtypes. Clin Cancer Res. 2008;14:5158-65.

12. Wirapati P, Sotiriou C, Kunkel S, Farmer P, Pradervand S, Haibe-Kains B, et al. Meta-analysis of gene expression profiles in breast cancer: toward a unified understanding of breast cancer subtyping and prognosis signatures. Breast Cancer Res. 2008;10:R65.

13. Ivshina AV, George J, Senko O, Mow B, Putti TC, Smeds J, et al. Genetic reclassification of histologic grade delineates new clinical subtypes of breast cancer. Cancer Res. 2006;66:10292-301.

14. Sotiriou C, Wirapati P, Loi S, Harris A, Fox S, Smeds J, et al. Gene expression profiling in breast cancer: understanding the molecular basis of histologic grade to improve prognosis. J Natl Cancer Inst. 2006;98:262-72.

15. Van't Veer LJ, Dai H, van deVijver MJ, He YD, Hart AA, Mao M, et al. Gene expression profiling predicts clinicaloutcome of breast cancer. Nature. 2002;415:530-6.

16. van de Vijver MJ, He YD, van't Veer LJ, Dai H, Hart AA, Voskuil DW, et al. A gene-expression signature as a predictor of survival in breast cancer. N Engl J Med. 2002;347:1999-2009.

17. Wang Y, Klijn JG, Zhang Y, Sieuwerts AM, Look MP, Yang F, et al. Geneexpression profiles to predict distant metastasis of lymph-node-negative primary breast cancer. Lancet. 2005:365:671-9.

18. Paik S, Shak S, Tang G, Kim C, Baker J, Cronin M, et al. A multigene assay to predict recurrence of tamoxifen-treated, node negative breast cancer. $\mathrm{N}$ Engl J Med. 2004;351:2817-26.

19. Ma XJ, Wang Z, Ryan PD, Isakoff SJ, Barmettler A, Fuller A, et al. A two gene expression ratio predicts clinical outcome in breast cancer patients treated with tamoxifen. Cancer Cell. 2004:5:607-16.

20. Finak G, Bertos N, Pepin F, Sadekova S, Souleimanova M, Zhao H, et al. Stromal gene expression predicts clinical outcome in breast cancer. Nat Med. 2008;14:518-27.

21. Teschendorff AE, Miremadi A, Pinder SE, Ellis IO, Caldas C. An immune response gene expression module identifies a good prognosis subtype in estrogen receptor negative breast cancer. Genome Biol. 2007;8:R157.

22. Guyon I, Weston J, Barnhill S, Vapnik V. Gene selection for cancer classification using support vector machines. Machine Learning. 2002:46:389-422.

23. Kononenko I. Estimating attributes: analysis and extensions of RELIEF. Proc European Conf Machine Learning. 1994;784:171-82.

24. Li Y, Lu BL. Feature selection based on loss-margin of nearest neighbor classification. Pattern Recognit. 2009;42:1914-21.

25. Thawonmas R, Abe $S$. A novel approach to feature selection based on analysis of class region. IEEE Trans Syst Man and Cybern. 1997;27:196-207.

26. Guyon I, Elisseeff A. An introduction to variable and feature selection. J Mach Learn Res. 2003:3:1157-82.

27. Kohavi R, John GH. Wrapper for feature subset selection. Artif Intell. 1997:97:273-324.

28. Hua J, Tembe W, Dougherty ER. Feature selection in the classification of high-dimension data, in: IEEE International Work shop on Genomic Signal Processing and Statistics, 2008, doi:10.1109/GENSIPS.2008.4555665. 
29. Jin X, Xu A, Bie R, Guo P. Machine learning techniques and chi-square feature selection for cancer classification using SAGE gene expression profiles. Lect Notes Comput Sci. 2006;3916:106-15.

30. Liao C, Li S, Luo Z. Gene selection using Wilcoxon rank sum test and support vector machine for cancer. Lect Notes Comput Sci. 2007:4456:57-66.

31. Biesiada J, Duch W. Feature selection for high-dimensional data-a Pearson redundancy based filter. Adv Soft Comput. 2008;45:242-9.

32. Rocchi $L$, Chiari $L$, Cappello A. Feature selection of stabile-metric parameters based on principal component analysis. Med Biol Eng Comput. 2004;42:71-9.

33. Gheyas I, Simith L. Feature subset selection in large dimensionality domains. Pattern Recognit. 2010;43:5-13.

34. Yang J, Honavar V. Feature subset selection using a genetic algorithm. IEEE Intell Sys App. 1998;13:44-9.

35. Vieira SM, Sousa MC, Runkler TA. Ant colony optimization applied to feature selection in fuzzy classifiers. Lect Notes Comput Sci. 2007;4529:778-88.

36. Ronen $M$, Jacob Z. Using simulated annealing to optimize feature selection problem in marketing applications. Eur J Oper Res. 2006;171:842-58.

37. Tan F, Fu X, Wang H, Zhang Y, Bourgeois A. A hybrid feature selection approach for microarray gene expression data. Lect Notes Comput Sci. 2006;3992:678-85.

38. Yan Z, Yuan C. Ant colony optimization for feature selection in face recognition. Lect Notes Comput Sci. 2004;3072:221-6.

39. Osei-Bryson KM, Giles K, Kositanurit B. Exploration of a hybrid feature selection algorithm. J Oper Res Soc. 2004;54:790-7.

40. Mao KZ. Feature subset selection for support vector machines through discriminative pruning analysis. IEEE Trans Syst Man Cybern B Cybern. 2004;34:60-7.

41. Zadeh LA. Fuzzy sets. Inform Control. 1965;8(3):338-53.

42. Jensen $R$, Shen Q. Fuzzy-rough sets assisted attribute selection. IEEE Trans Fuzzy Syst. 2007;15:73-89.

43. Kovalerchuk B, Triantaphyllou E, Ruiz JF, Clayton J. Fuzzy logic in computeraided breast cancer diagnosis: analysis of lobulation. Artif Intell Med. 1997;11(1):75-85

44. Gonzalez A, Perez R. Selection of relevant features in a fuzzy genetic learning algorithms. IEEE Trans Syst Man Cybern. 2002;33:417-27.

45. Peña-Reyes CA, Sipper M. A fuzzy-genetic approach to breast cancer diagnosis. Artif Intell Med. 1999;17:131-55.

46. Lee H-M, Chen C-M, Chen J-M, Jou Y-L. An efficient fuzzy classifier with feature selection based on fuzzy entropy. IEEE Trans Syst Man Cybern. 2001;31:426-32

47. Li Y, Wu ZF. Fuzzy feature selection based on min-max learning rule and extension matrix. Pattern Recognit. 2008;41:217-26.

48. Marcelloni F. Feature selection based on a modified fuzzy C-means algorithm with supervision. Info Sci. 2003;151:201-26.

49. Rezaee MR, Goedhart B, Lelieveldt BPF, Reiber JHC. Fuzzy feature selection. Pattern Recognit. 1999;32:2011-9.

50. Nascimento S. Fuzzy Clustering via Proportional Membership Mode Amsterdam. The Netherlands: IOS Press; 2005. Frontiers in Artificial Intelligence and Applications.

51. Haibe-Kains B, Desmedt C, Rothé F, Piccart M, Sotiriou C, Bontempi G. A fuzzy gene expression-based computational approach improves breast cancer prognostication. Genome Biol. 2010;11:R18.

52. Javier Lopez F, Cuadros M, Blanco A, Concha A. Unveiling Fuzzy Associations between Breast Cancer Prognostic Factors and Gene Expression Data, DEXA'09. 20th International Workshop on Database and Expert Systems Application. 2009 pp.338-342.

53. Hedjazi L, Aguilar-Martin J, Le Lann M-V, Kempowsky T, et al. Towards a unified principle for reasoning about heterogeneous data: a fuzzy logic framework. Int J Unc Fuzz Knowl Based Syst. 2012;20:281-302.

54. Kaplan EL, Meier P. Nonparametric estimation from incomplete observations. J Am Stat Assoc. 1958;53:457-81.

55. McGovern SL, Qi Y, Pusztai L, Symmans WF, Buchholz TA. Centromere protein-A, an essential centromere protein, is a prognostic marker for relapse in estrogen receptor-positive breast cancer. Breast Cancer Res. 2012;14:R72. PubMed: 22559056.

56. Jain AK, Murty MN, Flynn PJ. Data clustering: a review. J ACM Comput Surv. 1999;31(3):264-323.

57. Dubois D, Prade $H$. The three semantics of fuzzy sets. Fuzzy Sets Syst. 1997;90:141-50.

58. Medasani S, Kim J. An overview of membership function generation techniques for pattern recognition. Int J Approx Reason. 1998;19:391-417.
59. Hedjazi L, Aguilar-Martin J, Le Lann M-V. Similarity-margin based feature selection for symbolic interval data. Pattern Recognit Lett. 2011;32(4):578-85.

60. Hedjazi L, Aguilar-Martin J, Le Lann M-V, Kempowsky T. Membership-Margin based Feature Selection for Mixed-Type and High-Dimensional Data. 2013. submitted to: Information Sciences 2013 Manuscript Number: INS-D-12-491.

61. Aguilar J, Lopez R, De M. The process of classification and learning the meaning of linguistic descriptions of concepts. In: Gupta MM, Sanchez E, editors. Approximate reasoning in decision analysis. Amsterdam: North Holland Publishing Company; 1982. p. 165-75.

62. Aguilar Martin J, Martin M, Piera N. Conceptual connectivity analysis by means of fuzzy partitions. Uncertainty in knowledge bases. Lect Notes Comput Sci. 1991;521:165-72.

63. Hedjazi L. A Tool for cancer diagnosis/prognosis based on information extracted from clinical databases and microarray analysis. PhD manuscript, Toulouse, France, December $8^{\text {th }}$ 2011. http://tel.archives-ouvertes.fr/tel00657959/fr/.

64. Aguado JC, Aguilar-Martin J. A mixed qualitative-quantitative self-learning classification technique applied to diagnosis. QR'99 The Thirteenth International Workshop on Qualitative Reasoning. Chris Price. 1999, 124-128.

65. Wessels LFA, Reinders MJT, Hart AAM, Veenman CJ, Dai H, He YD, et al. A protocol for building and evaluating predictors of disease state based on microarray data. Bioinformatics. 2005;21:3755-62.

\section{Submit your next manuscript to BioMed Central and take full advantage of:}

- Convenient online submission

- Thorough peer review

- No space constraints or color figure charges

- Immediate publication on acceptance

- Inclusion in PubMed, CAS, Scopus and Google Scholar

- Research which is freely available for redistribution 